\title{
Superficial spreading squamous cell carcinoma of the uterine cervix involving the endometrium: Report of two cases with emphasis on the likely molecular mechanism
}

\author{
MITSUAKI ISHIDA and HIDETOSHI OKABE \\ Department of Clinical Laboratory Medicine and Division of Diagnostic Pathology, \\ Shiga University of Medical Science, Shiga, Japan
}

Received June 29, 2012; Accepted September 20, 2012

DOI: $10.3892 / \mathrm{ol} .2012 .953$

\begin{abstract}
Squamous cell carcinoma (SCC) of the cervix generally invades directly into the uterine wall, but in rare cases it spreads superficially to the inner surface of the uterus, thereby replacing the endometrium. This type is called superficial spreading SCC. In the present study we report two cases of this unusual form of cervical SCC and discuss the possible molecular mechanism involved. Two females, aged 64 (case 1) and 59 (case 2) years old, presented with post-menopausal vaginal bleeding. Histopathological studies of the resected specimens revealed non-keratinizing SCC of the cervix (pTIIA1 in case 1 and pTIIB in case 2). A notable finding in the two tumors was that atypical squamous cells were extending and replacing the endometrium directly from the cervix. Glandular involvement of atrophic endometrial glands was observed, however no invasive growth was observed in the endometrium in either case. Immunohistochemical studies revealed that CD138 was strongly expressed in the carcinoma cells superficially spreading in the endometrium in the two cases. It has been reported that the loss of expression of CD138, a cell-surface heparan sulfate proteoglycan participating in cell-cell and cell-extracellular matrix interactions, is correlated with tumor invasion and progression to cervical SCC. Our present findings suggest that CD138 expression in carcinoma cells may participate in superficial spread by regulating cell-cell interactions.
\end{abstract}

\section{Introduction}

Squamous cell carcinoma (SCC) is the most common malignant tumor of the cervix, comprising $70-80 \%$ of cervical

Correspondence to: Dr Mitsuaki Ishida, Department of Clinical Laboratory Medicine and Division of Diagnostic Pathology, Shiga University of Medical Science, Tsukinowa-cho, Seta, Otsu, Shiga 520-2192, Japan

E-mail: mitsuaki@belle.shiga-med.ac.jp

Key words: uterine cervix, superficial spreading squamous cell carcinoma, CD138 malignancies. Cervical SCC generally invades directly into the uterine wall with or without parametrial involvement. Cervical SCC that spreads superficially to the inner surface of the uterus and replaces the endometrium with carcinoma cells is called superficial spreading SCC and has been extremely rarely reported (1-4). In the present study, we report two additional cases of superficial spreading SCC of the cervix involving the endometrium and discuss the possible molecular mechanism of this unusual spreading form of cervical SCC. This study was approved by the Ethics Committee of Shiga, University of Medical Science, Shiga, Japan. Consent was obtained from the patients.

\section{Patients and methods}

\section{Case reports}

Case 1. A 64-year-old Japanese female presented with persistent post-menopausal vaginal bleeding. Cervical biopsy at a gynecological clinic revealed invasive SCC and the patient was referred to Shiga University of Medical Science Hospital. The patient was pre-operatively classified as clinical stage IIA1 since vaginal examination revealed vaginal involvement of the cervical cancer and the tumor size was $1 \mathrm{~cm}$. The patient underwent total hysterectomy and bilateral salpingooophorectomy.

The post-operative course has been uneventful, and the patient has been free from tumor recurrence or metastasis for 10 months.

Case 2. A 59-year-old Japanese female presented with persistent post-menopausal vaginal bleeding. Cervical smear at a gynecological clinic revealed presence of a high-grade squamous intraepithelial lesion and the patient was referred to Shiga University of Medical Science Hospital. Magnetic resonance imaging revealed a cervical tumor, measuring $\sim 4 \times 4 \mathrm{~cm}$, directly invading into the parametrium. Histopathological study of the cervical biopsy specimen showed invasive SCC. The patient was pre-operatively classified as clinical stage IIB and underwent total hysterectomy and bilateral salpingooophorectomy.

The post-operative course has been uneventful, and the patient has been free from tumor recurrence or metastasis for 6 months. 



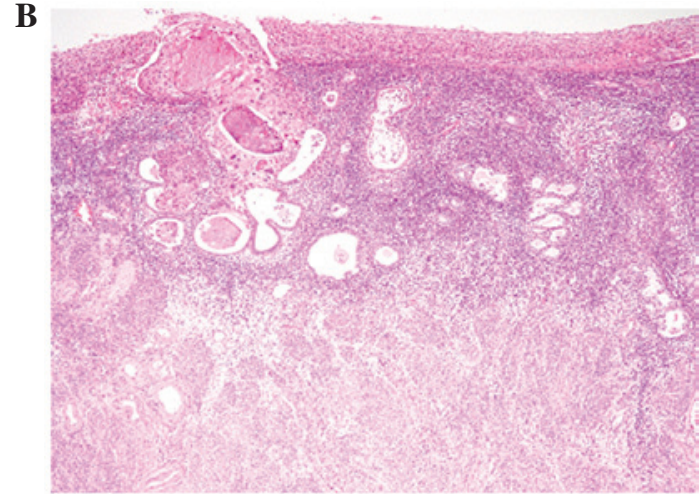

D



Figure 1. Histopathological and immunohistochemical features of case 1. (A) Mild invasive growth of atypical squamous cells in the cervix (hematoxylin and eosin, x100). (B) Low-power view of superficial spreading atypical squamous cells in the endometrium (hematoxylin and eosin, x100). (C) High-power view showing superficial spreading and glandular involvement of atypical squamous cells (hematoxylin and eosin, x200). (D) CD138 was strongly expressed in the neoplastic squamous cells spreading in the endometrium (x200). Mildly invasive squamous cells in the cervix also expressed CD138 (inset, x100).

A

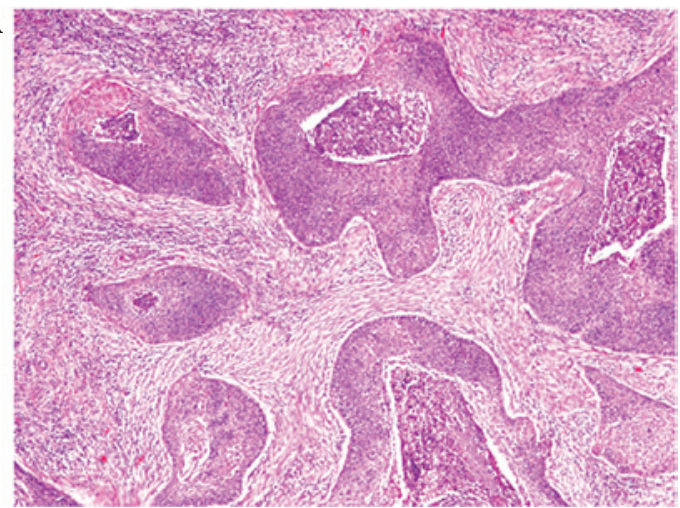

C



B

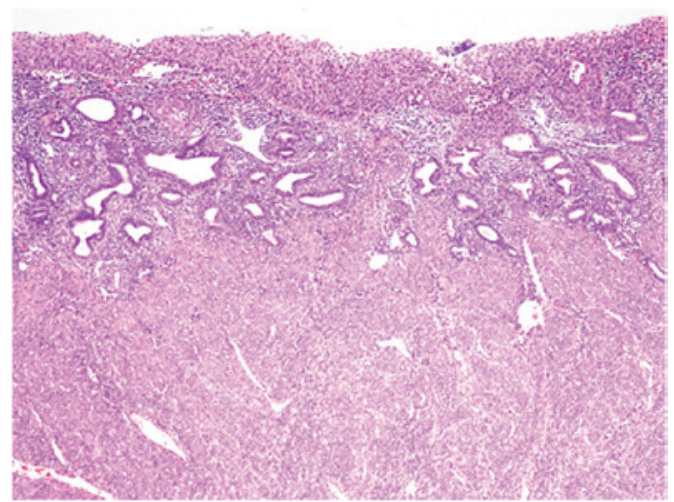

D

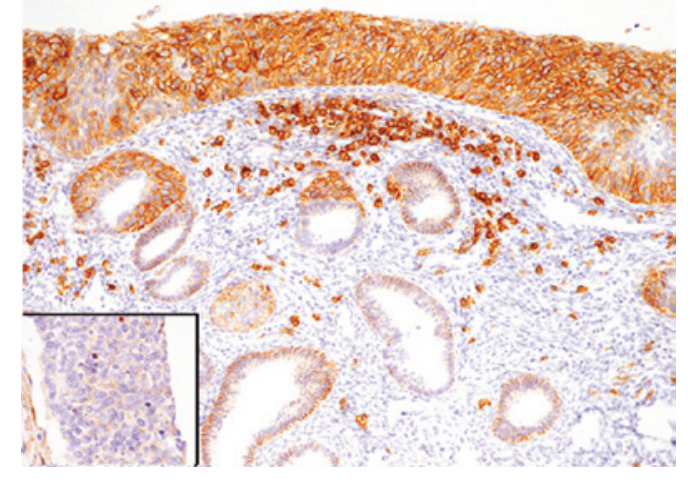

Figure 2. Histopathological and immunohistochemical features of case 2. (A) Invasive growth of squamous cell carcinoma with desmoplastic reaction in the cervix (hematoxylin and eosin, x100). (B) Low-power view of superficial spreading atypical squamous cells in the endometrium (hematoxylin and eosin, x100). (C) High-power view showing superficial spreading and glandular involvement of atypical squamous cells (hematoxylin and eosin, $\mathrm{x} 200$ ). (D) CD138 was strongly expressed in the atypical squamous cells spreading in the endometrium. Plasma cells were also positive for CD138 in the endometrium (x200). No CD138 expression was observed in the invasive squamous cell carcinoma cells in the cervix (inset, $\mathrm{x} 200$ ). 
Materials and methods. The formalin-fixed, paraffinembedded tissue blocks of the resected specimens were cut into 3- $\mu \mathrm{m}$-thick sections, deparaffinized and rehydrated. Each section was stained with hematoxylin and eosin and then used for immunostaining. Immunohistochemical analyses were performed using an autostainer (XT system Benchmark, Ventana Medical System, Tucson, AZ, USA) according to the manufacturer's instructions. A mouse monoclonal antibody against CD138 (syndecan-1; B-A38, Cell Marque, Rocklin, CA, USA) was used.

\section{Results}

Case 1. Proliferation of atypical squamous cells with large oval nuclei and a single nucleolus was observed in the entire squamous epithelium of the cervix and mitotic figures were noted in the upper portion of the squamous epithelium. Most of the cervical tumor was composed of this intraepithelial lesion with glandular involvement, however, focal superficial invasive growth was noted (Fig. 1A). Keratinization was not evident. Intraepithelial involvement and superficial invasive growth of carcinoma cells in the vagina were also observed. According to these findings, a diagnosis of non-keratinizing SCC of the cervix (pTIIA1) was made.

The notable finding of the present case was that atypical squamous cells were extending and replacing the endometrium directly from the cervix (Fig. 1B). Glandular involvement of atrophic endometrial glands was observed (Fig. 1B and C), but no invasive growth was noted in the endometrium. Bilateral tubes and ovaries were free from carcinoma spread. In addition, no lymph node metastasis was detected.

Immunohistochemically, all the carcinoma cells that were spreading superficially in the endometrium (Fig. 1D), in the mildly invasive lesion of the cervix and vagina (Fig. 1D, inset) and in the intraepithelial lesions of the cervix and vagina were diffusely and strongly positive for CD138.

Case 2. Invasive neoplastic growth composed of irregular islands or large nests was observed in the entire cervix (Fig. 2A). Neoplastic cells had large slightly eosinophilic cytoplasm and large oval nuclei with a conspicuous nucleolus. Intercellular bridges and individual cell keratinization were observed, however, keratinizaion was not evident and keratin pearls were not observed. The tumor had directly invaded into the parametrium, but not into the vagina. Therefore, a diagnosis of non-keratinizing SCC of the cervix (pTIIB) was made.

Neoplastic squamous cells were extending and replacing the endometrium directly from the cervix (Fig. 2B). Glandular involvement of the atrophic endometrial glands was observed (Fig. 2B and C), but no myometrial invasion was noted. The entire endometrium was replaced by carcinoma cells, however, bilateral tubes and ovaries were free from carcinoma spread. In addition, no lymph node metastasis was detected.

Immunohistochemical analyses revealed that CD138 was strongly and diffusely expressed in the neoplastic squamous cells superficially spreading in the endometrium (Fig. 2D) and the intraepithelial lesion of the cervix, however CD138 expression was hardly observed in the neoplastic squamous cells of the invasive lesion of the cervix (Fig. 2D, inset).

\section{Discussion}

In the present study, we described two additional cases of superficial spreading SCC of the cervix involving the endometrium. The clinicopathological features of the 26 previously reported cases of superficial spreading SCC of the cervix and the two present cases are as follows: i) the age of all patients was over 50 years (average, 61.9 years; range, 52-78 years; information on the patients' age was available in 14 cases); ii) the most common clinical presentation was genital bleeding; and iii) Fallopian tube with or without ovarian involvement was present in 9 cases (1-4).

Kushima et al reported five cases of superficial spreading SCC of the cervix involving the endometrium and/or Fallopian tube and ovary (3). They concluded that most tumors of this type were monoclonal neoplasia originating from the cervical mucosa with subsequent superficial spreading to the upper genital mucosa according to loss of heterozygosity analyses (3). The two present cases were also thought to be of cervical mucosa origin that had spread superficially in the endometrium.

The molecular mechanism of the superficial spreading of carcinoma cells is not well understood. CD138, also known as syndecan-1, is a cell-surface heparan sulfate proteoglycan and participates in cell-cell and cell-extracellular matrix interactions (5). CD138 is expressed in normal epithelial cells, including stratified squamous epithelium, as well as plasma cells. A correlation between CD138 expression and carcinogenesis has been investigated in various organs. Increased expression of CD138 has been reported in endometrial and ovarian carcinomas $(6,7)$. By contrast, decreased expression of CD138 has been reported to correlate with tumor invasion and progression of cervical cancer (8-10). Shinyo et al reported that the loss of CD138 expression is an early event in cervical carcinogenesis, as this molecule is strongly expressed in cervical intraepithelial neoplasia (CIN) I and II. However, its expression is reduced in CIN III, and CD138 expression in microinvasive and invasive SCC is lower compared with CIN III (9). In the two present cases, CD138 was strongly expressed in the intraepithelial lesions of the cervix as well as in the cells that were superficially spreading in the endometrium. Therefore, the findings of the present cases suggest that CD138 expression in carcinoma cells may participate in superficial spreading by regulating cell-cell interactions and, by contrast, carcinoma cells lacking CD138 expression show overt invasive growth.

\section{References}

1. GungorT, Altinkaya SO,OzatM,Akbay S and Mollamahmutoglu L: Unusual form of superficial spreading squamous cell carcinoma of cervix involving the endometrium, bilateral tubes and ovaries: a case report with literature review. Arch Gynecol Obstet 283: 323-327, 2011.

2. Tan GC, Isa MR, Ng SP and Jamil MA: Unusual form of superficial spreading microinvasive squamous cell carcinoma of uterine cervix involving the endometrium of uterus. J Obstet Gynaecol Res 30: 363-367, 2004.

3. Kushima M, Fujii H, Murakami K, et al: Simultaneous squamous cell carcinomas of the uterine cervix and upper genital tract: loss of heterozygosity analysis demonstrates clonal neoplasms of cervical origin. Int J Gynecol Pathol 20: 353-358, 2001. 
4. Pins MR, Young RH, Crum CP, Leach IH and Scully RE: Cervical squamous cell carcinoma in situ with intraepithelial extension to the upper genital tract and invasion of tubes and ovaries: report of a case with human papilloma virus analysis. Int J Gynecol Pathol 16: 272-278, 1997.

5. Bernfield M, Götte M, Park PW, et al: Functions of cell surface heparan sulfate proteoglycans. Ann Rev Biochem 68: 729-777, 1999.

6. Oh JH, Kim JH, Ahn HJ, et al: Syndecan-1 enhances the endometrial cancer invasion by modulating matrix metalloproteinase-9 expression through nuclear factor kappa B. Gynecol Oncol 114: 509-515, 2009.

7. Davies EJ, Blackhall FH, Shanks JH, et al: Distribution and clinical significance of heparan sulfate proteoglycans in ovarian cancer. Clin Cancer Res 10: 5178-5186, 2004.
8. Numa F, Hirabayashi K, Kawasaki K, et al: Syndecan-1 expression in cancer of the uterine cervix: association with lymph node metastasis. Int J Oncol 20: 39-43, 2002.

9. Shinyo Y, Kodama J, Hasengaowa, Kusumoto T and Hiramatsu Y: Loss of cell-surface heparan sulfate expression in both cervical intraepithelial neoplasm and invasive cervical cancer. Gynecol Oncol 96: 776-783, 2005.

10. Kim YI, Lee A, Lee BH and Kim SY: Prognostic significance of syndecan-1 expression in cervical cancers. J Gynecol Oncol 22: 161-167, 2001. 